\title{
MERGING OF ELLIPTICAL GALAXIES AS A POSSIBLE ORIGIN OF THE INTERGALACTIC STELLAR POPULATION
}

\author{
Letizia Stanghellini \\ National Optical Astronomy Observatory, 950 North Cherry Avenue, Tucson, AZ 85719; 1stanghellini@noao.edu \\ AND \\ A. Cesar González-García and Arturo Manchado \\ Instituto de Astrofísica de Canarias, vía Láctea s/n, La Laguna, E-38200 Tenerife, Spain; cesar.gonzalez@iac.es, amt@iac.es \\ Received 2005 October 6; accepted 2006 March 2
}

\begin{abstract}
We present $N$-body simulations of elliptical galaxy encounters into dry mergers to study the resulting unbound intergalactic stellar population, in particular that of the post-main-sequence stars. The systems studied are pairs of spherical galaxies without dark halos. The stellar content of the model galaxies is distributed into mass bins representing low- and intermediate-mass stars $\left(0.85-8 M_{\odot}\right)$ according to the Salpeter initial mass function. Our models follow the dynamical evolution of galaxy encounters colliding head-on from initial low-energy parabolic or high-energy mildly hyperbolic orbits and for a choice of initial mass ratios. The merging models with initial parabolic orbits have $M_{2} / M_{1}=1$ and 10 , and they leave behind, respectively, $5.5 \%$ and $10 \%$ of the total initial mass as unbound stellar mass. The merging model with an initial hyperbolic orbit has $M_{2} / M_{1}=1$ and leaves behind $21 \%$ of its initial stellar mass as unbound mass, showing that the efficiency in producing intergalactic stars through a high-energy hyperbolic encounter is about 4 times that of a parabolic encounter of the same initial mass ratio. By assuming that all progenitor galaxies, as well as the merger remnants, are homologous systems we find that the intergalactic starlight is $17 \%$ and $28 \%$ of the total starlight, respectively, for the parabolic and hyperbolic encounters with $M_{2} / M_{1}=1$. In all models, stars of different mass have the same probability of becoming unbound and feeding the intergalactic stellar population.
\end{abstract}

Subject headings: galaxies: elliptical and lenticular, $\mathrm{cD}$ - galaxies: interactions — galaxies: stellar content — planetary nebulae: general - stars: AGB and post-AGB

\section{INTRODUCTION}

Intergalactic (IG) starlight, both diffuse and from resolved stars, has been intensively studied in the past decade in the IG medium of both groups and clusters of galaxies. The long-lived populous class of low- and intermediate-mass stars, in the form of red giants (red giant branch [RGB] and asymptotic giant branch [AGB]), planetary nebulae (PNe), and their diffuse light, has been observed in the IG medium of different types of galaxy associations, from poor groups such as the M81 group of galaxies (Feldmeier et al. 2004b) and the Leo region corresponding to the $\mathrm{H}$ I cloud (Castro-Rodríguez et al. 2003), to compact tidal groups (White et al. 2003), up to the Virgo (Ferguson et al. 1998; Durrell et al. 2002; Feldmeier et al. 1998, 2003, 2004a) and Coma (Gerhard et al. 2005) Clusters, as well as in higher redshift clusters (Zibetti et al. 2005).

The observations tell us that a considerable fraction of postmain-sequence (PMS) stars in galaxy associations are found between galaxies. The contribution of the unbound stars to the total mass and light of the association varies greatly, depending mainly on the galaxy concentration of the considered cluster or group. Observations of poorly populated groups seem to indicate that the upper limit of the IG contribution to the total light is very low (up to $1.6 \%$; Castro-Rodríguez et al. 2003), while surveys of IG PNe, $\mathrm{AGB}$, and RGB stars in the nearby clusters indicate that the intracluster (IC) starlight contribution is roughly $5 \%-20 \%$ of the total starlight (Durrell et al. 2002; Aguerri et al. 2005; Feldmeier et al. 2004a), depending on the assumptions made about the stellar populations probed and on the completeness of the sample surveyed. The observed range of the fraction of IG starlight is also supported by the observations of diffuse starlight in clusters at $z \sim 0.25$ (Zibetti et al. 2005).

A host of explanations for the origin of the IG starlight have been proposed in a variety of studies. The tidal interactions between galaxies have been explored in some depth by several authors, among them Merritt (1983) and Moore et al. (1996). An alternative scenario that is well suited to the IC environment has been proposed by Muccione \& Ciotti (2004); in their model the stellar stripping from galaxies is driven by interactions between the stellar orbits within the galaxies and the cluster tidal field. Among all possible explanations for the existence of the IG starlight, the scenario of elliptical galaxy merging (dry merging) has been the least explored.

In this paper we present numerical models of dry merging of galaxies with different initial mass ratios, with the aim of describing possible scenarios for the production of the unbound stellar mass in the IG medium. Our models are crafted in particular to account for the PMS IG population produced by the merging of elliptical galaxy pairs. The red galaxy merging scenario may not be very common at present in the cores of galaxy clusters, where the velocity dispersions are of the order of $1000 \mathrm{~km} \mathrm{~s}^{-1}$. Nonetheless, red mergers have very recently proved to be the common evolutionary path to field (van Dokkum 2005) and cluster (Tran et al. 2005) elliptical galaxies and may be also the path to produce IG starlight in galaxy groups or in cluster periphery, where the velocity dispersions are typically much lower than in young galaxy clusters cores (Arnaboldi et al. 2004). Dry mergers have recently been studied by González-García \& van Albada (2005a, 2000b) and have proved to preserve the properties of the elliptical galaxy fundamental plane (González-García \& van Albada 2003; Nipoti 
et al. 2003), providing a further tool to study mergers and stellar populations.

In $\S 2$ we present our models, including the type of numerical experiment performed, the astrophysical input parameters, the considerations for stellar populations, the initial conditions and constraints, and a description of the methodology and stability tests of the numerical models. Section 3 illustrates our results, with particular attention to the IG stellar population produced. The discussion is in $\S 4$, where we present a limited comparison with the observational data and the likelihood that the drymerging scenario might account for the observed IG starlight. This paper represents a first attempt at modeling the IG population with dry merging of galaxies, and the parameter space is thus exploratory. More detailed models, and a larger array of calculated observable parameters, will be presented in forthcoming papers.

\section{MODELS}

\subsection{Galaxies, Initial Conditions}

We perform numerical models of three cases of dry mergers, with a choice of initial mass ratios $M_{2} / M_{1}=1$ or $10 .^{1}$ The initial galaxy conditions are similar to those described by GonzálezGarcía \& van Albada (2005a), where a systematic study of the encounters between two spherical systems without a dark matter halo was performed. These models without dark matter are a good first approximation to study the IG population in galaxy associations. In fact, González-García \& van Albada (2005b) have shown that merging of elliptical galaxies with dark halos produces a luminous particle distribution that is very similar to that resulting from the merging of elliptical galaxies without dark matter (González-García \& van Albada 2005a). On the other hand, models similar to those presented here, but with the inclusion of dark matter, will be performed in the future to confirm our results, since González-García \& van Albada (2005a, 2005b) did not compare the unbound stellar population derived from the merging processes with and without the inclusion of dark matter.

We use isotropic spherical (Jaffe 1983) models as initial conditions for our experiments, and the algorithm developed by P. A. H. Smulders \& M. Balcells (1995, unpublished; see GonzálezGarcía \& van Albada 2005a for a detailed description of the algorithm). The projected surface mass density (hereafter, surface density) of such models presents a slope that decreases roughly as $R^{1 / 4}$, which makes it a suitable representation for elliptical galaxies, although the central parts present a cusp. The distribution function (DF) presents an analytical solution for the collisionless Boltzmann equation, allowing the implementation of $\mathrm{N}$-body initial conditions.

In Table 1 we summarize the characteristics of the initial galaxy pairs. Column (1) gives the run identification code, where the number indicates the mass ratio, the lower case letter denotes the impact parameter ( $h$ defines the head-on impact), and the capital letter indicates the energy of the orbit $(P$ for parabolic and $Z$ for zero energy at infinity, or hyperbolic orbit). Hereafter, we will identify the encounter models with this code. Column (2) gives the initial mass ratio of the colliding galaxies, column (3) gives their initial separation, column (4) gives their initial relative velocity, and columns (5) and (6) give, respectively, the impact parameter and the orbital energy of the initial setup.

In Table 1 and later in describing the model parameters we use the model units unless otherwise noted. We adopt nondimen-

\footnotetext{
1 In this paper the subscript " 1 " always refers to the less massive galaxy of the merging pair.
}

TABLE 1

Model Input, Galaxy Pair Parameters

\begin{tabular}{|c|c|c|c|c|c|}
\hline $\begin{array}{c}\text { Run } \\
\text { (1) }\end{array}$ & $\begin{array}{c}M_{2} / M_{1} \\
\text { (2) }\end{array}$ & $\begin{array}{l}r_{i} \\
(3)\end{array}$ & $\begin{array}{c}v_{i} \\
(4)\end{array}$ & $\begin{array}{c}b \\
(5)\end{array}$ & $\begin{array}{c}E_{\text {orb }} \\
(6)\end{array}$ \\
\hline $1 h P$. & 1 & 40 & 0.316 & 0 & 0 \\
\hline$\ldots \ldots \ldots \ldots \ldots \ldots$ & 10 & 61.62 & 0.604 & 0 & 0 \\
\hline 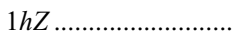 & 1 & 40 & 1.048 & 0 & 0.250 \\
\hline
\end{tabular}

sional units with Newton's constant of gravity $G=1$. In each run, the theoretical half-mass radius of the Jaffe model, $r_{\mathrm{J}}$, and the total mass of the less massive galaxy, $M_{1}$, are also set to 1 . The models may be compared with real galaxies using the following scaling:

$$
\begin{gathered}
{[M]=M_{\mathrm{J}}=4 \times 10^{11} M_{\odot},} \\
{[R]=r_{\mathrm{J}}=10 \mathrm{kpc},} \\
{[t]=2.4 \times 10^{7} \mathrm{yr} .}
\end{gathered}
$$

By adopting these units, the velocity unit is

$$
[v]=414 \mathrm{~km} \mathrm{~s}^{-1} \text {. }
$$

Following the Jaffe (1983) notation, the mass inside a radius $r$ is defined as

$$
M(r)=\frac{r}{r+r_{\mathrm{J}}} M
$$

In run $10 h P$ the galaxy model with mass $M_{2}$ is a scaled-up version of the model with mass $M_{1}$ with 10 times more particles, constructed following the scaling relation between mass and radius given by Fish (1964):

$$
\frac{M_{1}}{R_{1}^{2}}=\frac{M_{2}}{R_{2}^{2}}=K,
$$

where $K$ is a constant. Following Jaffe's definition and Fish's relation, the theoretical half-mass radius for the more massive galaxy is 3.162 .

In order to limit the calculation time and yet preserve the physical significance of the results, we modify Jaffe's models in such a way as to obtain working galaxy models with finite radii. We impose a cutoff radius to all galaxy models, with $R_{1}=10$ for the less massive galaxies. Such a radius includes only $91 \%$ of the mass in the theoretical Jaffe model; thus, we need to rescale the half-mass radius to keep $M_{1}=1$. The rescaled half-mass radius for the less massive galaxy is then equal to 0.82 .

Model $1 h P$ is an equal-mass encounter between two galaxies on a parabolic orbit, where $M_{1}=M_{2}=1$. The centers of the two galaxies are placed at an initial distance of $4 R_{1}$. Model $10 \mathrm{hP}$ is an encounter between two galaxies with a mass ratio 10 on a radial parabolic encounter, initially placed at a distance $3 R_{1}+R_{2}$, where $R_{1}$ and $R_{2}$ are the galaxy radii of the working models. Model $1 \mathrm{hZ}$ is an encounter between two galaxies with $M_{1}=M_{2}=1$, placed initially on a mildly hyperbolic orbit.

The choices of initial conditions for the galaxies are adequate to represent the observed mergers that occur in elliptical galaxies. The galaxy mass ratios chosen for the simulations, $M_{2} / M_{1}=1$ and 10 , are the extremes of the observed merging galaxy sample by van Dokkum (2005). 
TABLE 2

Model Input, Stellar Parameters

\begin{tabular}{|c|c|c|c|c|}
\hline $\begin{array}{c}\text { Bin } \\
\left(M_{\odot}\right) \\
(1)\end{array}$ & $\begin{array}{c}M \\
\left(M_{\odot}\right) \\
(2)\end{array}$ & $\begin{array}{l}\Phi_{\text {bin }} \\
\text { (3) }\end{array}$ & $\begin{array}{c}N_{\text {particles }} \\
\text { (4) }\end{array}$ & $\begin{array}{c}m_{\text {particle }} \\
\text { (5) }\end{array}$ \\
\hline $0.85-1.4 \ldots \ldots \ldots \ldots \ldots \ldots$ & 1 & 0.5151 & 156060 & $3.3 \times 10^{-6}$ \\
\hline $1.4-3.0 \ldots \ldots \ldots \ldots \ldots \ldots \ldots$ & 2 & 0.3443 & 52121 & $6.6 \times 10^{-6}$ \\
\hline $3.0-8.0 \ldots \ldots \ldots \ldots \ldots$ & 6 & 0.1406 & 7050 & $2.0 \times 10^{-5}$ \\
\hline 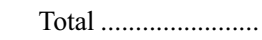 & & 1 & 215231 & 1 \\
\hline
\end{tabular}

\subsection{Stellar Components}

We populate our model galaxies with model stars that represent stellar masses in the $m=0.85-8 M_{\odot}$ mass range, ${ }^{2}$ whose progeny includes RGB, AGB, and PNe. Since the main scope of our modeling is to study the stellar population that produce PMS stars, we neglect all stars outside this mass range. In order to model these stars we use test particles whose masses are proportional to the stellar masses they represent. We assume the validity of the Salpeter (1955) initial mass function (IMF), $\Psi(m) \propto$ $m^{-2.35}$, and consider three representative mass bins for the progenitors of massive (3-8 $M_{\odot}$ ), intermediate $\left(1.4-3 M_{\odot}\right)$, and low-mass $\left(0.85-1.4 M_{\odot}\right)$ PMS stars. The population of each mass bin corresponds to the integration of the Salpeter mass function in that bin, scaled to the entire population considered and ignoring stars outside the mass range. We then calculate the mass fraction for each bin as

$$
\Phi_{\mathrm{bin}}=A^{-1} \int_{m_{\min }}^{m_{\max }} m \Psi(m) d m,
$$

where $m_{\min }$ and $m_{\max }$ are the limits of the mass bin considered and

$$
A=\int_{0.85}^{8.0} \Psi(m) d m=0.8777
$$

is the normalization of the Salpeter law in the entire mass range.

To characterize the mass bins in the $N$-body simulation, we use a representative mass for each bin $\left(1,2\right.$, and $\left.6 M_{\odot}\right)$. The model stars in the model galaxy with $M=1$ have such masses that, after accounting for the Salpeter IMF, the total galaxy mass is equal to unity.

In Table 2 we summarize the characteristics of the stellar population in the $M=1$ galaxies. Column (1) gives the mass bin in solar masses, column (2) gives the representative mass in that bin, column (3) gives the mass fraction in that mass bin, column (4) gives the number of particles in the bin, and column (5) gives the (dimensionless) stellar mass for each particle in that bin. Note that the mass of each particle is different from the mass of the star it represents, but the ratio between particle masses in different bins is the same as the ratio of the representative masses in that bin. This is a good representation, since we are interested in relative results for the mass bins. In runs $1 h P$ and $1 h Z$ both galaxies have number of particles per mass bin as in Table 2. The least massive galaxy of run $10 \mathrm{hP}$ also has stellar population as in Table 2, while the more massive galaxy has an initial setup with 10 times more particles in each mass bin, but with the same mass per particle.

\footnotetext{
${ }^{2}$ Hereafter, $m$ refers to the stellar mass, to distinguish it from $M$, the galaxy
}

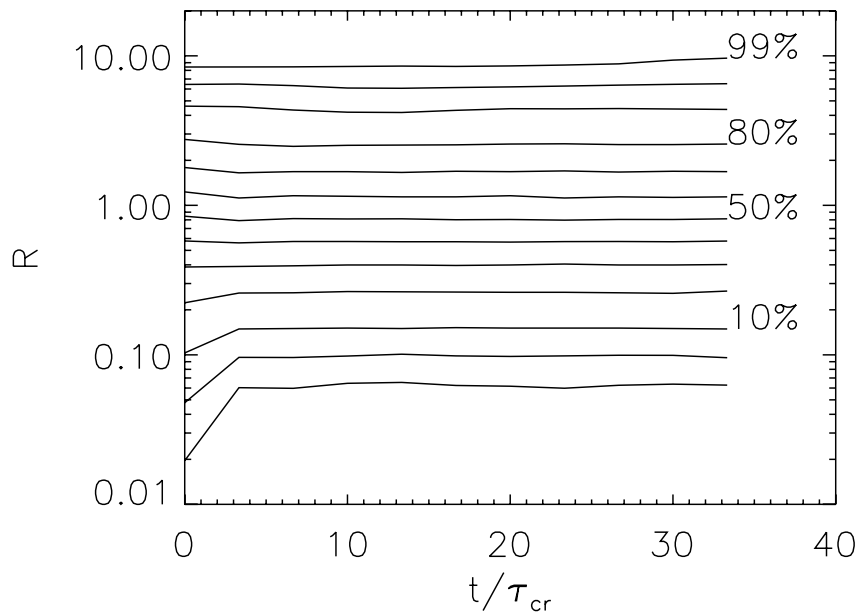

FIg. 1.-Test run to check the stability of our initial models. The plot shows the evolution of the mass inside different radii. The top line gives the $99 \%$ mass radius, while the third line from the bottom gives the $10 \%$ mass radius.

The initial distribution of the populations of particles with different mass is such that each stellar population follows the same Jaffe law. In this way, there are fewer particles from the high-mass bin at any given radius. We use the DF as in González-García \& van Albada (2005a), extending it for each mass bin.

\subsection{Integration Method and Stability}

We have used the parallel tree code GADGET-1 (Springel et al. 2001) on the Beowulf cluster at the Instituto de Astrofísica de Canarias (IAC), where a typical run on 16 CPUs takes of the order of $1.5 \times 10^{5} \mathrm{~s}$. Gravitational Plummer softening (see, e.g., Binney \& Tremaine 1987, eq. [2-194], p. 747) was set to $\sim 1 / 10$ of the half-mass radius of the less massive galaxy, with softening parameter $\varepsilon=0.075$. The tolerance parameter (see Barnes \& Hut 1986) was set to $\theta=0.8$. Quadrupole terms were included in the force calculation. GADGET-1 uses a variable time step $\left(t_{s}\right)$, and we choose $t_{s} \propto 1 /|a|^{0.5}$, where $a$ is the particle acceleration. We set the maximum time step to $1 / 100$ of the half-mass crossing time, and the minimum time step to zero.

We have checked the stability of our input initial model for 33 crossing times. In Figure 1 we show the results from a test run that has been used to check the stability of our models. There, the evolution of the mass inside different radii has been followed at different mass fraction levels, showing stability in a large range of $t / \tau_{\mathrm{cr}}$, where $\tau_{\mathrm{cr}}$ is the half-mass crossing time. The test shows that the system relaxes for about 4 crossing times and remains stable thereafter. This initial relaxation is probably due to the presence of the particle softening in the code.

Models were evolved for at least 10 dynamical crossing times of the merged system after merging, to allow the system to relax (reach virialization). Conservation of energy is sound in all the runs, with variations lower than $0.5 \%$ of the total energy.

We performed an additional run of a model identical to $1 \mathrm{hP}$, except with twice as many particles in each mass bin. Our merging simulation on this additional model results in an IG mass fraction of $5.22 \%, 5.24 \%$, and $5.34 \%$ for particles in the first, second, and third mass bin, respectively, proving that our results are stable against mass resolution.

\section{RESULTS}

The three models of dry merging presented in this paper have different initial conditions, both in the mass content of the parent 
TABLE 3

Model Results

\begin{tabular}{|c|c|c|c|}
\hline $\begin{array}{c}\text { Bin } \\
\left(M_{\odot}\right) \\
(1)\end{array}$ & $\begin{array}{c}M_{\mathrm{IG}} \\
(\%) \\
(2)\end{array}$ & $\begin{array}{c}L_{\mathrm{IG}} / L_{\text {tot }} \\
(\%) \\
(3)\end{array}$ & $\begin{array}{c}L_{\mathrm{IG}} / L_{m} \\
(\%) \\
(4)\end{array}$ \\
\hline \multicolumn{4}{|c|}{$1 h P$} \\
\hline $0.85-1.4 \ldots \ldots \ldots \ldots \ldots$ & 5.53 & & \\
\hline $1.4-3.0 \ldots \ldots \ldots \ldots \ldots . .$. & 5.55 & & \\
\hline $3.0-8.0 \ldots \ldots \ldots \ldots \ldots \ldots .$. & 5.37 & & \\
\hline Total ........................... & 5.48 & 17 & 20 \\
\hline \multicolumn{4}{|c|}{$10 h P$} \\
\hline $0.85-1.4 \ldots \ldots \ldots \ldots \ldots \ldots \ldots$ & 9.87 & & \\
\hline $1.4-3.0 \ldots \ldots \ldots \ldots \ldots \ldots$ & 10.20 & & \\
\hline $3.0-8.0 \ldots \ldots \ldots \ldots \ldots \ldots . .$. & 9.57 & & \\
\hline Total ............................ & 9.97 & 14 & 17 \\
\hline \multicolumn{4}{|c|}{$1 h Z$} \\
\hline $0.85-1.4 \ldots \ldots \ldots \ldots \ldots \ldots$ & 20.6 & & \\
\hline $1.4-3.0 \ldots \ldots \ldots \ldots \ldots \ldots . .$. & 20.6 & & \\
\hline $3.0-8.0 \ldots \ldots \ldots \ldots \ldots \ldots . .$. & 20.9 & & \\
\hline Total ........................... & 20.7 & 28 & 38 \\
\hline
\end{tabular}

galaxies and in their relative velocities, as described in $\S 2$. As model $1 h P$ is allowed to evolve, the system passes through pericenter for the first time at $t=85$, when an exchange of orbital energy into internal energy occurs. The particles having initial binding energy close to zero gain enough energy at this stage to be expelled from the system and become unbound particles. The two galaxies finally meet again 30 time units after the first encounter. This is the time when the actual merging occurs. New particles are able to become unbound at this time, as a consequence of new particle encounters and exchange of energy. The merging time is $2.8 \times 10^{9} \mathrm{yr}$ after the initial placement in orbit, with the unit convention given in $\S 2.1$. A very similar situation occurs in model $10 \mathrm{hP}$, where the two unequal galaxies meet at pericenter after $t=70$ since the initial placement in orbit, then meet again 131 time units after the first encounter, with merging time of $4.82 \times 10^{9} \mathrm{yr}$. The galaxies of model $1 \mathrm{hZ}$, initially in mildly hyperbolic orbits, meet for the first time at $t=34.5$, and after 65.5 time units the second and final encounter occurs, ending in the merging episode. The merging time for $1 \mathrm{hZ}$ is then $2.4 \times 10^{9} \mathrm{yr}$. All runs are stopped after 10 merging crossing times, after virialization has been reached.

In Table 3 we give the characteristics of the stellar populations of our models after the merging has occurred. We give the run code, the mass bin (col. [1]), and the fraction of resulting unbound mass (col. [2]). We also give the unbound starlight fractions in columns (3) and (4), as described in $\S 4$.

By inspecting the data of Table 3 we find several interesting results from our merging simulations. First, we see that for all runs the percentage of particles that become unbound does not depend on the mass bin. This result is expected, since the particle expulsion is due to gravitational acceleration, and indicates that if we were able to observe all IG stars with the same probability, we should recover the IMF of the original galaxies. Second, the final fraction of unbound mass is different for the three merging models. In $1 h P$ the fraction of unbound to total mass is $M_{\mathrm{IG}} / M_{\mathrm{tot}}=$ $(5.48 \pm 0.1) \times 10^{-2}$, which means that $\sim 95 \%$ of the total initial mass still remains in the merger $\left(M_{m}=1.89\right.$, with $\left.M_{\text {tot }}=2\right)$. In run $10 \mathrm{hP}$ the fraction of unbound to total mass is almost twice as high, with $M_{\mathrm{IG}} / M_{\mathrm{tot}}=(9.97 \pm 0.4) \times 10^{-2}$, corresponding to a merger with $90 \%$ of the initial mass $\left(M_{m}=9.9\right.$ for $\left.M_{\mathrm{tot}}=11\right)$. Naturally, the larger fraction of unbound mass in model $10 \mathrm{hP}$ compared to $1 h P$ could be imputed to the initial mass ratio, but it may also be the result of the high kinetic energy due to the higher initial relative velocity in model $10 h P$. The third model, $1 \mathrm{hZ}$, has the highest efficiency in producing unbound stars. The IG stellar mass in this case represents $21 \%$ of the total initial mass, and the merger retains only $79 \%$ of the initial mass. In this case, the merger has $M_{m}=1.58$ from an initial total mass $M_{\mathrm{tot}}=2$.

In Figures 2, 3, and 4 we show the location of the model stars in the radius-binding energy plane for each of the mass bins, for the $1 h P, 10 h P$, and $1 h Z$ models, respectively. Both $R$ and $E_{\text {bind }}$ in the plot are dimensionless variables, $R$ being the distance from the merger's center. Note that the plots do not include the complete range where particles are found, but rather the range where most particles are found. For example, out to a radius $R=50$ we find $90 \%$ of the $1 h \mathrm{~h}$ particles and up to $99 \%$ of the $1 \mathrm{hP}$ particles. These figures give a clear idea of the nature of the merger and of the distribution of bound and unbound particles for each mass bin in each model considered. Unbound particles are found out to a distance of $R_{\max } \approx 160,600$, and 200 , respectively, for
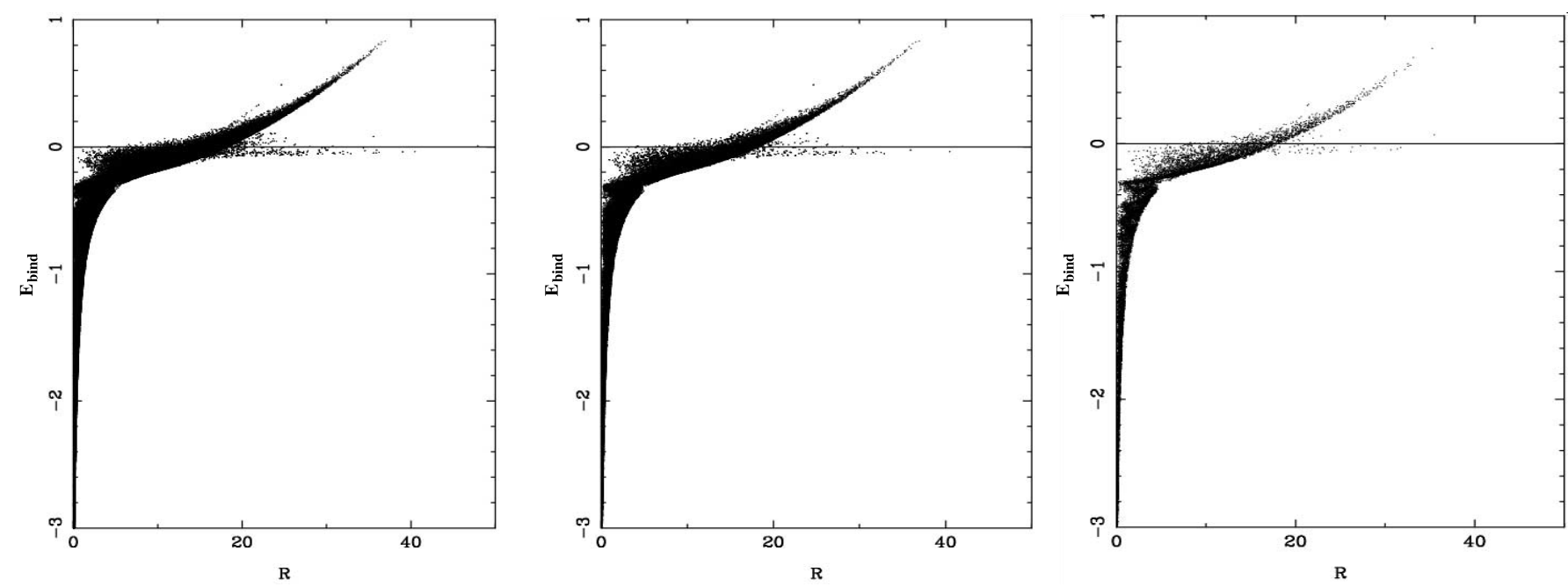

FIG. 2.-Binding energy vs. distance (from the merger's center) of the model stars in the $1 h P$ model. Left to right: Low, intermediate, and high-mass bins. 

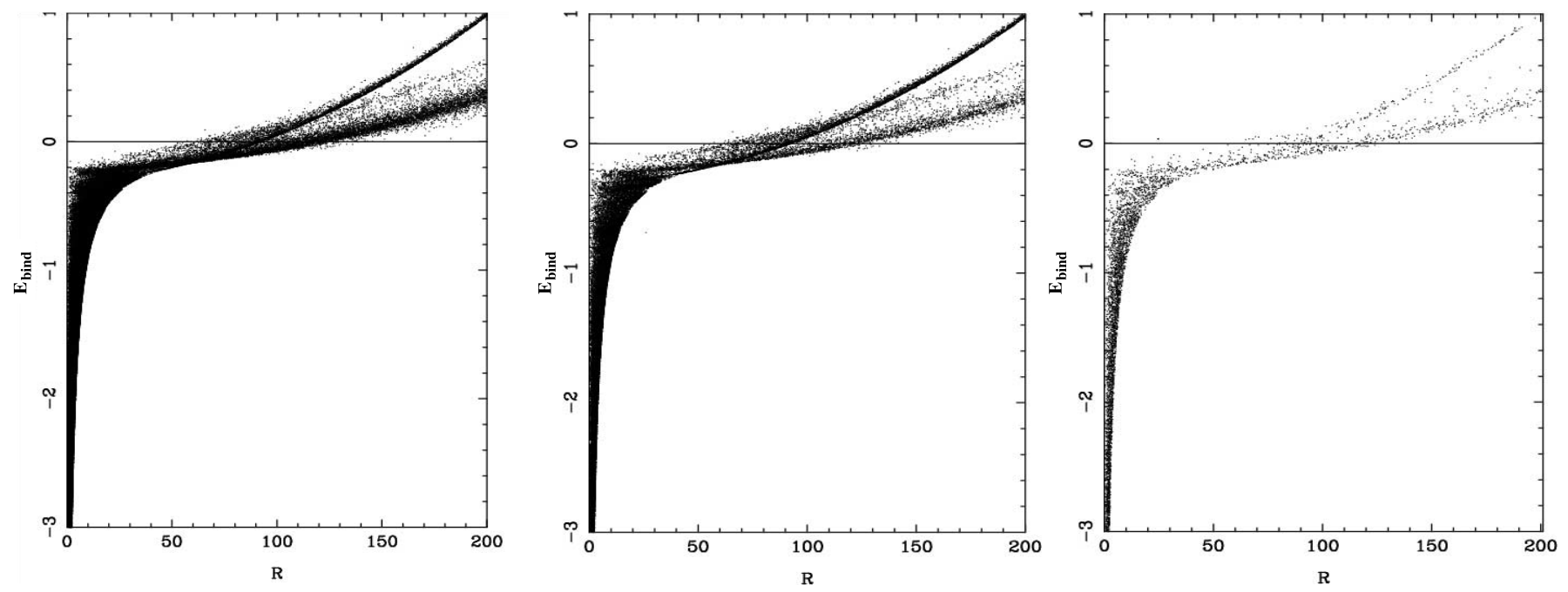

FIG. 3.-Same as in Fig. 2, but for the $10 h P$ model.

models $1 h P, 10 h P$, and $1 h Z$. We do find a mild anticorrelation between the particle mass and $R_{\max }$. By running a model similar to $1 h P$, but with twice as many particles per mass bin, we proved that the anticorrelation is due to the relative populations of the bins, and it does not have physical significance.

In Figure 5, 6, and 7 we plot the surface density calculated within annuli of increasingly larger radii from the merger's center in models $1 h P, 10 h P$, and $1 h Z$, respectively. In these figures we plot the logarithm of the surface density $\mu$ against $R^{1 / 4}$, where $R$ is the distance from the merger's center. The three lines represent the different mass bins of stellar populations. Note that these plots are not cumulative and that the surface density values have been evaluated in concentric annuli equally spaced in $\log R$. This representation is useful to show where the galaxy merger profile dominates and where the de Vaucouleurs (1959) profiles start to be perturbed by the unbound particles. By comparing the $1 \mathrm{hP}$ (Fig. 5) and $1 \mathrm{hZ}$ (Fig. 7) models, we see that the surface density profile is similarly perturbed, but that the IG component outside the $R^{1 / 4}=2$ annulus is much more important in the $1 h Z$ than in the $1 h P$ model. The de Vaucouleurs' slope is affected by the IG particles outmost of $R^{1 / 4} \sim 2.5$ in the $10 h P$ model (Fig. 6). In all models, test particles from the different mass bins contribute to the merger and the IG mass in very similar fashion.

\section{DISCUSSION}

Elliptical galaxy encounters were modeled with $N$-body simulation, and they produce mergers in a fraction of the Hubble time. During the process they expel a fraction of their stellar content. The model stars expelled continue to evolve in the IG medium, eventually going through the red giant, AGB, and postAGB phases. The fraction of unbound mass resulting through this process depends on the properties of the encounter. By comparing the two models with the initial parabolic orbits and different mass ratios we conclude that the model with the higher mass ratio produces a larger fraction of unbound mass with respect to the total (as well as the merger's) mass. By comparing the $M_{2} / M_{1}=1$ runs we see that the initial hyperbolic orbit results in a unbound mass fraction that is almost 4 times that of the parabolic orbit encounter.

Since the elliptical mergers belong to the fundamental plane of elliptical galaxies (González-García \& van Albada 2003) in the same way as for the two original merging galaxies, we can
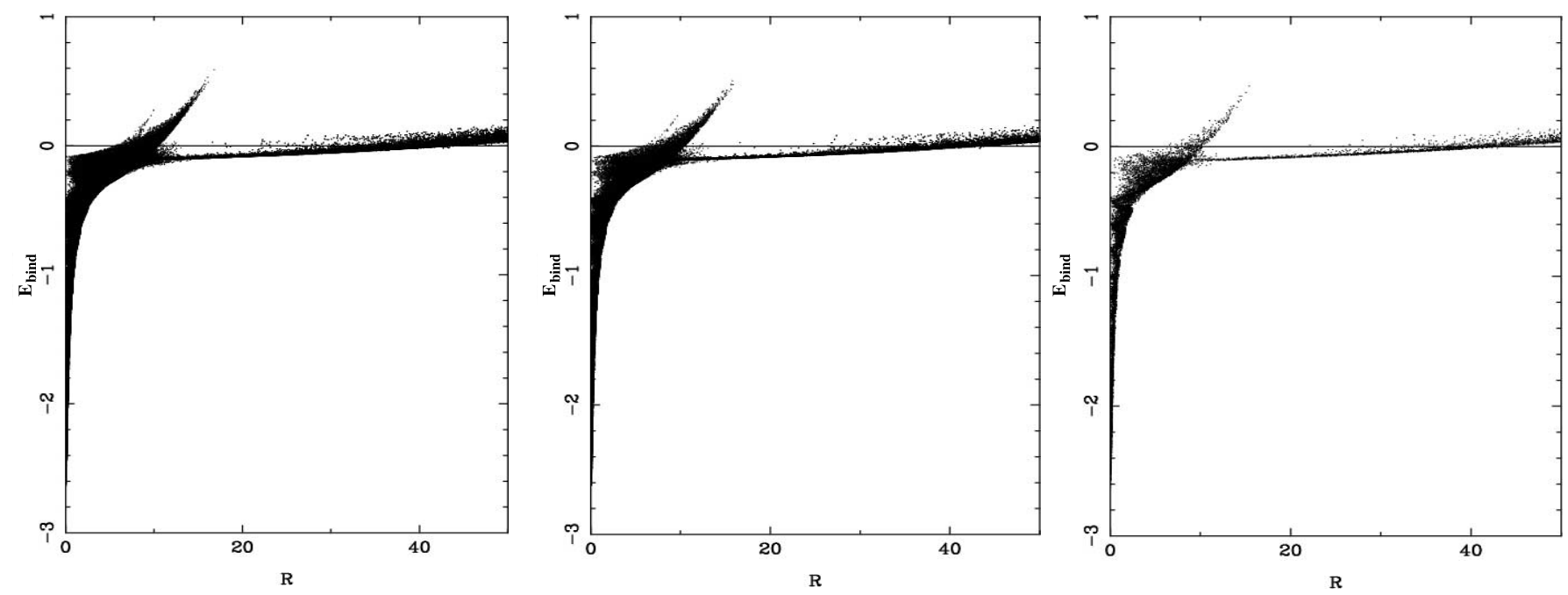

FIG. 4.-Same as in Fig. 2, but for the $1 h Z$ model. 


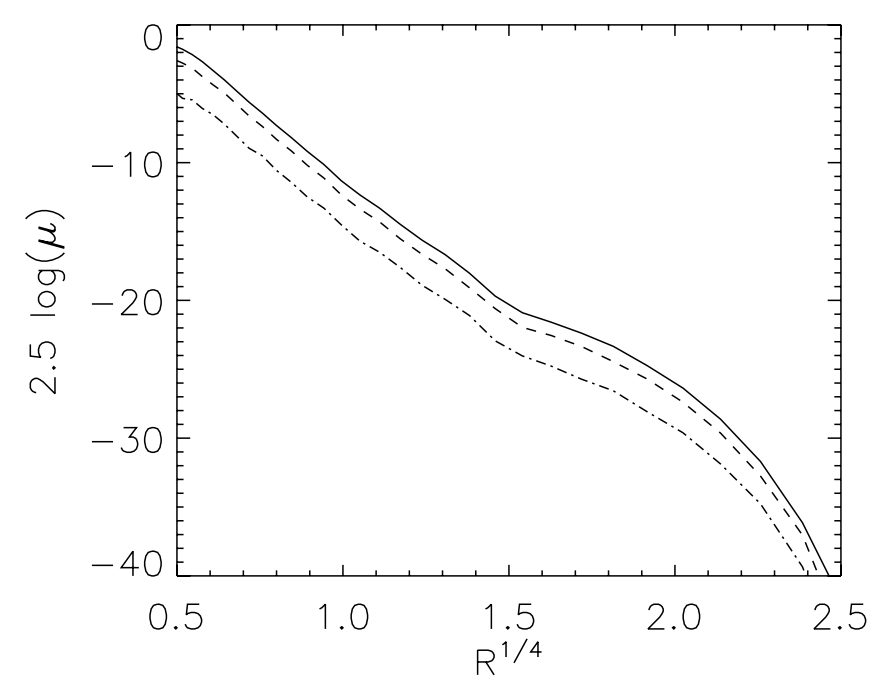

FIG. 5.-Surface density vs. $R^{1 / 4}$ in model $1 h P$. The three lines represent the mass bins, where the solid line represents the low-mass bin, the dashed line the intermediate-mass bin, and the dash-dotted line the high-mass bin.

determine the fraction of unbound-to-total starlight for all our models simply by assuming that both the initial galaxies and the final mergers are homologous galaxies. If we assume that every galaxy of the merging pairs and all mergers obey the massluminosity relation $M / L \propto M^{0.2}$ (Jorgensen et al. 1996), we can calculate $L_{\mathrm{IG}} / L_{\mathrm{tot}}$, the ratio of the IG starlight to total starlight. In dry-merging encounters the relation linking the total to individual galaxy luminosity can be written as $L_{\mathrm{IG}}=L_{1}+$ $L_{2}-L_{m}$. By using the mass fractions of unbound mass from Table 3 we found $\left[L_{\mathrm{IG}} / L_{\mathrm{tot}}\right]_{1 h P}=17 \%,\left[L_{\mathrm{IG}} / L_{\mathrm{tot}}\right]_{10 h P}=14 \%$, and $\left[L_{\mathrm{IG}} / L_{\mathrm{tot}}\right]_{1 h Z}=28 \%$. The fractions of the unbound to merger's starlight are, respectively, $\left[L_{\mathrm{IG}} / L_{m}\right]_{1 h P}=20 \%,\left[L_{\mathrm{IG}} / L_{m}\right]_{10 h P}=$ $17 \%$, and $\left[L_{\mathrm{IG}} / L_{m}\right]_{1 h Z}=38 \%$ (see also Table 3 ).

By recalling that our model galaxies are populated by lowand intermediate-mass stars, their $L_{\mathrm{IG}} / L_{m}$ fractions may be compared with the observed PMS populations. The IG starlight ratios that we recover in our simulations are in broad agreement with the observations of several IC and intragroup stellar populations. Below, we examine a few cases.

The most studied case of IG stellar population is that of $\mathrm{PNe}$ in Virgo. The mechanism proposed in this paper is probably more likely to occur as a possible origin of the IC stellar population, or

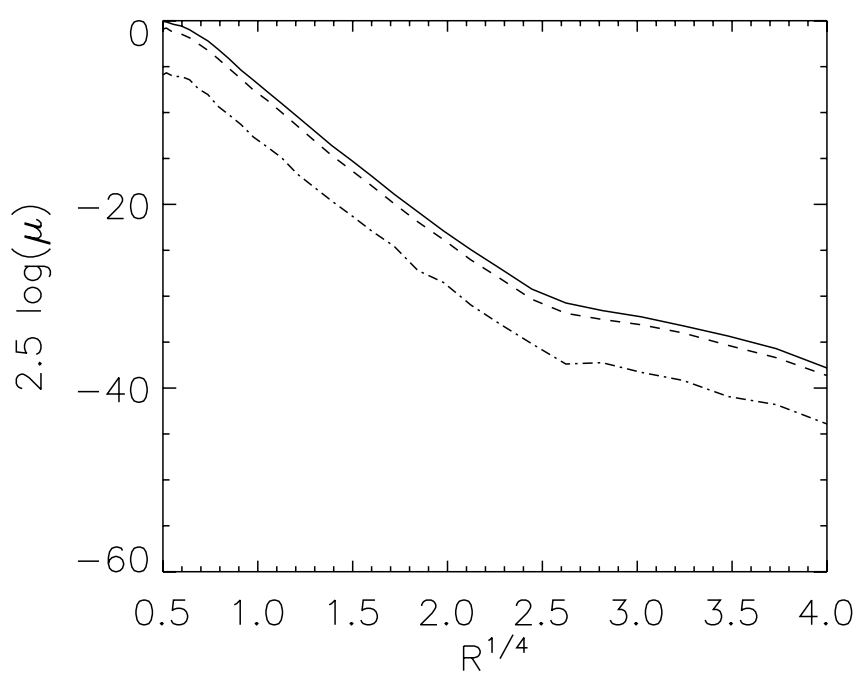

FIG. 6.-Same as in Fig. 5, but for model 10hP.

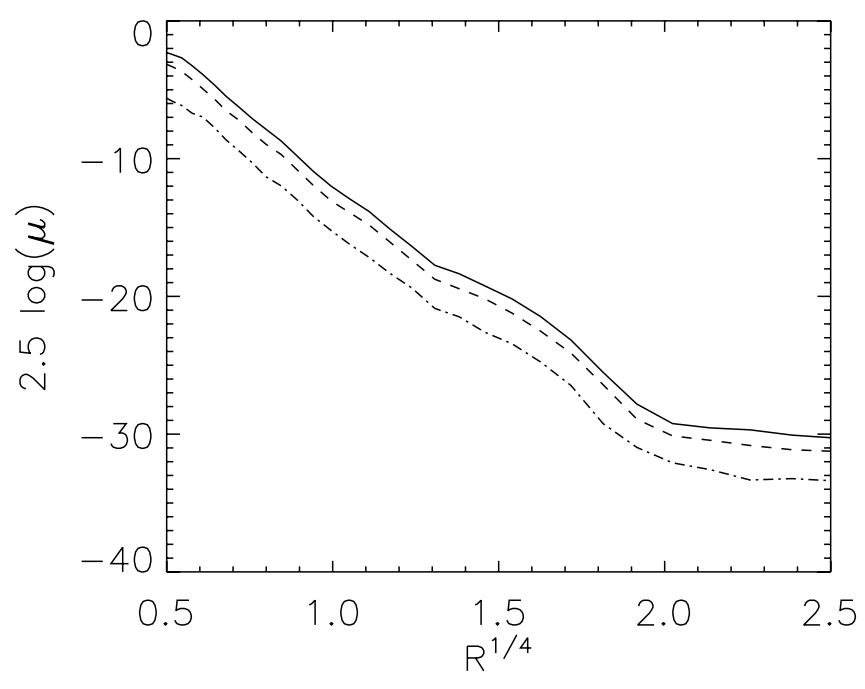

FIG. 7.-Same as in Fig. 5, but for model $1 \mathrm{hZ}$.

parts thereof, in the cluster periphery, rather than at the center of a cluster such as Virgo. Arnaboldi et al. (2004) have studied the velocity dispersion of a sample of Virgo IC PNe and found that several fields have dispersion velocities much lower $\left(247 \mathrm{~km} \mathrm{~s}^{-1}\right)$ than the canonical Virgo dispersion of $800 \mathrm{~km} \mathrm{~s}^{-1}$, obviously a consequence of the fact that the cluster is young and highly nonuniform. Feldmeier et al. (2004a) found that $\sim 16 \%$ of the starlight in the Virgo Cluster is in the IG medium, independent of the location within Virgo, and this number is encompassed by our results.

Nonetheless, the observed ratio of unbound to total starlight in the Virgo Cluster derived from PNe must be used "cum grano salis." To derive this ratio from observations, one must evaluate the theoretical luminosity-specific PN density, $\alpha_{\mathrm{PN}}=B t_{\mathrm{PN}}$ (Renzini $\&$ Buzzoni 1986), which is based on the fuel consumption theorem for PMS stars. The question is whether this theorem is adequate to describe a nebular population, where $t_{\mathrm{PN}}$, the PN lifetime, is not a stellar evolutionary time, determined by fuel consumption, but rather a timescale depending on the hydrodynamic evolution and the photoionization on the nebulae. While $t_{\mathrm{PN}}=25,000 \mathrm{yr}$ is typically adopted, the correct lifetime for PNe to be observable at high luminosity is probably much lower, as the hydrodynamic models by Villaver \& Stanghellini (2005) have shown. Furthermore, $t_{\mathrm{PN}}$ has not been parameterized for the mass and chemistry of the progenitor stars. Feldmeier et al. (2004a) advise using $\alpha_{\text {stars }}$ from other stellar sources, such as Virgo red giants (Durrell et al. 2002).

The red giant population of the Virgo IC, first observed by Ferguson et al. (1998), accounts for approximately $10 \%$ of the cluster (evolved) stellar mass. Ferguson et al. (1998) indicated that the IG population is likely to originate from elliptical and S0 galaxies, for their higher frequency in the cluster and their older stellar populations. IG starlight has been observed in Fornax, both in the form of PNe and other stars. Given the difficulty of PNe lifetime scaling, we prefer to use the results from IG nova observations (Neill et al. 2005), indicating that $\sim 16 \%-41 \%$ of the starlight in the Fornax cluster comes from IG stars. Both the Virgo and Fornax PMS IG stars might have a dry-merging origin, and these percentages are clearly in the range of our results (see Table 3, col. [4]). Group IG populations are typically evaluated to be 10 times lower than their cluster counterparts, although spectroscopic confirmation of, for example, the M81 PNe has not yet been published (Feldmeier et al. 2004b) and final counts are not available. 
The mere existence of low-mass PMS stars in a given stellar population requires a very old stellar population. Following Maraston's (1998) prescription, the turnoff mass in the first star mass bin corresponds to ages in the $\sim 4.8-22$ Gyr range. If, for the sake of argument, we assume that the progenitor galaxies were just formed at the time they were put in their relative orbits, none of their low-mass $\left(0.85-1.4 M_{\odot}\right)$ stars would have reached the turnoff by the time the merger is completely formed. More realistically, the merging galaxy pairs contain aging stellar populations when their first encounter occurs. Accordingly to van Dokkum and collaborators (van Dokkum et al. 1999; van Dokkum 2005), dry merging could have been important at intermediate redshifts when clusters were still assembling. Therefore, part of the IC stellar population may have been expelled at those cosmic times.

Zibetti et al. (2005) found that the IC light at large cluster radii is largely dominated by surface brightness excess around galaxies. From our Figure 5 we find that the IC contribution to the profile is above the $R^{1 / 4}$ law from $R^{1 / 4} \approx 1.5-2$. This implies that the IC stars contribute the most to the surface density out to $\approx 100 \mathrm{kpc}$ from the merger edge of model $1 \mathrm{hP}$ (or, with similar reasoning, about $60 \mathrm{kpc}$ from the merger edge in model $1 \mathrm{hZ}$ ) with the usual unit conversion, consistent with what observed by Zibetti et al. Naturally this analysis is sensitive to the choice of units that we use to compare the models with real galaxies, as described in $\S 2$, but it is worth showing that our equalmass encounter models are at least broadly consistent with the observations.
In this paper we have compared our models with the observed IG populations. In other words, we have implicitly assumed that all IG stars derive from dry merging and that all galaxies in a given observed cluster or group have gone through at least one merging process. In this extreme assumption the models correctly predict the observed IG starlight. Naturally, the case might well be that dry merging occurs only in a fraction of cases and that other mechanisms are at work in explaining the observed unbound starlight in galaxy associations. While the dry-merging scenario is certainly helpful to account for a fraction of the observed IG light, it may not work well near cluster centers and in high-velocity environments, where other mechanisms, such as mass stripping due to hyperbolic encounters, or disk galaxy merging, might be more efficient.

In the future, we plan to model encounters similar to those presented here, but including dark halos in elliptical galaxies. Furthermore, other phenomena related to dry merging and a larger range of initial conditions will be considered in future studies.

We warmly thank Luca Ciotti for important comments on the manuscript and for scientific discussions and Mark Dickinson and John Feldmeier for scientific discussion and bibliographic indications. L. S. thanks the European Southern Observatory and the IAC for their hospitality during the summers of 2004 and 2005, when this work was conceived and completed.

\section{REFERENCES}

Aguerri, J. A. L., Iglesias-Páramo, J., Vílchez, J. M., Muñoz-Tuñón, C., \& Sánchez-Janssen, R. 2005, AJ, 130, 475

Arnaboldi, M., Gerhard, O., Aguerri, J. A. L., Freeman, K. C., Napolitano, N. R., Okamura, S., \& Yasuda, N. 2004, ApJ, 614, L33

Barnes, J., \& Hut, P. 1986, Nature, 324, 446

Binney, J., \& Tremaine, S. 1987, Galactic Dynamics (Princeton: Princeton Univ. Press) Castro-Rodríguez, N., Aguerri, J. A. L., Arnaboldi, M., Gerhard, O., Freeman,

K. C., Napolitano, N. R., \& Capaccioli, M. 2003, A\&A, 405, 803

de Vaucouleurs, G. 1959, Handb. Phys., 53, 311

Durrell, P. R., Ciardullo, R., Feldmeier, J. J., Jacoby, G. H., \& Sigurdsson, S. 2002, ApJ, 570, 119

Feldmeier, J. J., Ciardullo, R., \& Jacoby, G. H. 1998, ApJ, 503, 109

Feldmeier, J. J., Ciardullo, R., Jacoby, G. H., \& Durrell, P. R. 2003, ApJS, 145, 65 2004a, ApJ, 615, 196

Feldmeier, J. J., Ciardullo, R., Jacoby, G. H., Durrell, P. R., \& Mihos, J. C. 2004b, in IAU Symp. 217, Recycling Intergalactic and Interstellar Matter, ed P.-A. Duc, J. Braine, \& E. Brinks (San Francisco: ASP), 64

Ferguson, H. C., Tanvir, N. R., \& von Hippel, T. 1998, Nature, 391, 461

Fish, R. A. 1964, ApJ, 139, 284

Gerhard, O., Arnaboldi, M., Freeman, K. C., Kashikawa, N., Okamura, S., \& Yasuda, N. 2005, ApJ, 621, L93

González-García, A. C., \& van Albada, T. S. 2003, MNRAS, 342, L36

- 2005a, MNRAS, 361, 1030 2005b, MNRAS, 361, 1043
Jaffe, W. 1983, MNRAS, 202, 995

Jorgensen, I., Franx, M., \& Kjaergaard, P. 1996, MNRAS, 280, 167

Maraston, C. 1998, MNRAS, 300, 872

Merritt, D. 1983, ApJ, 264, 24

Moore, B., Katz, N., Lake, G., Dressler, A., \& Oemler, A. 1996, Nature, 379, 613

Muccione, V., \& Ciotti, L. 2004, A\&A, 421, 583

Neill, J. D., Shara, M. M., \& Oegerle, W. R. 2005, ApJ, 618, 692

Nipoti, C., Londrillo, P., \& Ciotti, L. 2003, MNRAS, 342, 501

Renzini, A., \& Buzzoni, A. 1986, in Spectral Evolution of Galaxies (Dordrecht: Reidel), 195

Salpeter, E. E. 1955, ApJ, 121, 161

Springel, V., Yoshida, N., \& White, S. D. M. 2001, NewA, 6, 79

Tran, K.-V. H., van Dokkum, P., Franx, M., Illingworth, G. D., Kelson, D. D., \& Schreiber, N. M. F. 2005, ApJ, 627, L25

van Dokkum, P. G. 2005, AJ, 130, 2647

van Dokkum, P. G., Franx, M., Fabricant, D., Kelson, D. D., \& Illingworth, G. D. 1999, ApJ, 520, L95

Villaver, E., \& Stanghellini, L. 2005, ApJ, 632, 854

White, P. M., Bothun, G., Guerrero, M. A., West, M. J., \& Barkhouse, W. A. 2003, ApJ, 585, 739

Zibetti, S., White, S. D. M., Schneider, D. P., \& Brinkmann, J. 2005, MNRAS, 358, 949 